\title{
Submodular Maximization Meets Streaming: Matchings, Matroids, and More
}

\author{
Amit Chakrabarti* Sagar Kale* \\ $\{$ ac, sag\}@cs.dartmouth.edu
}

\begin{abstract}
We study the problem of finding a maximum matching in a graph given by an input stream listing its edges in some arbitrary order, where the quantity to be maximized is given by a monotone submodular function on subsets of edges. This problem, which we call maximum submodular-function matching (MSM), is a natural generalization of maximum weight matching (MWM), which is in turn a generalization of maximum cardinality matching (MCM). We give two incomparable algorithms for this problem with space usage falling in the semistreaming range-they store only $O(n)$ edges, using $O(n \log n)$ working memory - that achieve approximation ratios of 7.75 in a single pass and $(3+\varepsilon)$ in $O\left(\varepsilon^{-3}\right)$ passes respectively. The operations of these algorithms mimic those of Zelke's and McGregor's respective algorithms for MWM; the novelty lies in the analysis for the MSM setting. In fact we identify a general framework for MWM algorithms that allows this kind of adaptation to the broader setting of MSM.

In the sequel, we give generalizations of these results where the maximization is over "independent sets" in a very general sense. This generalization captures hypermatchings in hypergraphs as well as independence in the intersection of multiple matroids.
\end{abstract}

*Department of Computer Science, Dartmouth College. Supported in part by NSF grant CCF-1217375. 


\section{Introduction}

Maximum cardinality matchings and maximum weight matchings are basic concepts in graph theory and efficient algorithms for computing these structures are fundamental algorithmic results with myriad applications. The explosion of data-in particular graph data-over the past decade has motivated a number of researchers to revisit several algorithmic problems on graphs with a view towards designing space efficient algorithms that process their inputs in streaming fashion, i.e., via sequential access alone, though perhaps in multiple passes. In particular, a series of recent works [6,7,11,13, 18, 21] have studied the maximum cardinality matching (MCM) problem and its natural generalization, the maximum weight matching (MWM) problem, on graph streams.

In this work, we study a further generalization of MWM that we call the maximum submodularfunction matching problem or, more briefly, the maximum submodular matching (MSM) problem. This specific problem does not seem to have been studied in previous work, though there has been plenty of work in the optimization community on general (non-streaming) algorithms for constrained submodular function maximization under constraints more general than matchings (see, e.g., Feldman et al. [8] and the references therein, as well as our own discussion in Section 1.3). Our work gives the first results for the MSM problem in the data stream model. Our techniques in fact lead to results for a wider class of problems, including submodular maximization on hypermatchings and intersection of matroids.

Our study of MSM is inspired in part by its applicability to the Word Alignment Problem (WAP) from computational linguistics, as studied in Lin and Bilmes [17]: we are given a sourcelanguage string and a target-language string and the goal is to find a "good" mapping between their respective words. Lin and Bilmes [17] cast WAP as maximizing a suitable submodular function constrained to the intersection of two partition matroids (this is in turn closely related to bipartite matchings), and they note the improvement this gives over previous approaches that cast WAP as an instance of MWM.

A submodular function on a ground set $\mathcal{X}$ is defined to be a function $f: 2^{\mathcal{X}} \rightarrow \mathbb{R}$ that satisfies $f(A \cup B)+f(A \cap B) \leqslant f(A)+f(B)$ for all $A, B \subseteq \mathcal{X}$. For our purposes in this work, we will instead use the following "diminishing returns" characterization, which is well known to be equivalent to the definition just given: for all $Y \subseteq X \subset \mathcal{X}$ and $x \in \mathcal{X} \backslash X$, we have

$$
f(X \cup\{x\})-f(X) \leqslant f(Y \cup\{x\})-f(Y) .
$$

The function $f$ is said to be monotone if $f(Y) \leqslant f(X)$ whenever $Y \subseteq X \subseteq \mathcal{X}$ and proper if $f(\varnothing)=0$. An instance of MSM consists of a graph $G=(V, E)$ on vertex set $V=[n]:=\{1,2, \ldots, n\}$ and a non-negative monotone proper submodular function $f$ whose ground set is the edge set $E$, i.e., $f: 2^{E} \rightarrow \mathbb{R}_{+}$. The goal is to output a matching $M^{*} \subseteq E$ that maximizes $f\left(M^{*}\right)$; we shall refer to such a matching as an $f$-MSM of $G$. For a real number $\alpha \geqslant 1$, an $\alpha$-approximate $f$-MSM of $G$ is defined to be a matching $M \subseteq E$ such that $f(M) \geqslant \alpha^{-1} f\left(M^{*}\right)$.

A non-negative weight function $w: E \rightarrow \mathbb{R}_{+}$can be naturally extended to subsets of $E$ via $w(S)=\sum_{e \in S} w(e)$ for all $S \subseteq E$; the latter function $w$ is easily seen to be non-negative, monotone, proper, and submodular (it is in fact modular, a.k.a. linear). Therefore MSM generalizes the more famous MWM problem. Letting $w$ be a constant function gives us the even more special MCM problem. However, an important threshold is crossed in generalizing from MWM to MSM. The MWM problem is solvable in polynomial time [5, 10]-a monumental algorithmic triumph of the 20th century-whereas MSM hits an $\Omega(1)$ approximation threshold if it is to be solved in polynomial time; see Theorem 5 for a formal treatment.

Our concern in this paper is with graph streams: the input graph is described by a stream of edges $\{u, v\}$, with $u, v \in[n]$. We assume that the number of vertices, $n$, is known in advance 
and that each edge in $E$ appears exactly once in the input stream. The order of edge arrivals is arbitrary and possibly adversarial. We seek algorithms for MSM that use only quasi-linear working memory-i.e., $O\left(n(\log n)^{O(1)}\right)$ bits of storage, with $O(n \log n)$ being the holy grail—and process each edge arrival very quickly, ideally in $O(1)$ time. Algorithms with such guarantees have come to be known as semi-streaming algorithms [7]. Notice that $\Omega(n \log n)$ bits are necessary simply to store a matching that saturates $\Omega(n)$ vertices.

As with all optimization problems involving submodular functions, a study of MSM requires special care because the description of the submodular function $f$ needs $\Omega\left(2^{|E|}\right)$ space in general. Special cases do allow $f$ to be more compactly represented: such as MWM, where each edge in the stream arrives together with its weight. Since our goal is to give general algorithms, assuming no further structure for $f$, we will instead take the common approach of having $f$ specified by a value oracle that returns $f(S)$ when presented with $S \subseteq E$. See Section 2 for a precise explanation.

\subsection{Our Results}

We give two incomparable approximation algorithms for the MSM problem on graph streams, formally stated in the two theorems below. Both algorithms are semi-streaming: specifically, each stores only $O(n)$ edges, thereby using $O(n \log n)$ working memory 1 For brevity, "submodular $f$," means a non-negative monotone proper submodular function $f$, presented by a value oracle.

Theorem 1. For every submodular $f$, there is a one-pass semi-streaming algorithm that outputs a 7.75approximate $f$-MSM of an n-vertex input graph, storing at most $O(n)$ edges at all times.

Theorem 2. For every submodular $f$, and every constant $\varepsilon>0$, there is a multi-pass semi-streaming algorithm that makes $O\left(\varepsilon^{-3}\right)$ passes over an $n$-vertex graph stream and outputs a $(3+\varepsilon)$-approximate $f$ MSM of the graph. This algorithm stores only a matching in the input graph at all times; in particular it stores only $O(n)$ edges.

Perhaps more important than these specific approximation ratios is the technique behind these results. We identify a general framework for matching algorithms in graph streams. We show that whenever an MWM algorithm fits this framework, it can be adapted to the broader setting of MSM. The above theorems then follow by revisiting two recent MWM algorithms-that of Zelke [21] for Theorem 1 and that of McGregor [18] for Theorem 2-and showing that they fit our framework. Thus, the main contributions of our work are (1) the identification of the framework and (2) the novel analysis for the MSM setting.

Naturally, MWM algorithms base their actions on edge weights. A trivial way of giving a weight to an edge $e$ for the $f$-MSM problem is to use the quantity $f(\{e\})$. As may be expected, this is too naïve to be useful. Our first insight is that weights can be assigned to edges as they are encountered in the stream based on how much they improve the "current matching." Our second insight, specific to multi-pass algorithms, is that edge weights assigned this way can be calculated on each pass. Though the resulting weights may change from one pass to another, nevertheless, our framework and analysis technique allow us to recover a good approximation ratio.

Our framework is not deeply wedded to matchings: it is general enough to capture set maximization problems constrained to abstract "independent sets," for a very general notion of independence. Taking this view, we obtain two more families of results. The first applies to hypergraphs,

\footnotetext{
${ }^{1}$ Throughout the paper, we adopt the convention that edge weights in an MWM instance-and analogously, $f$ values of singletons in an MSM instance-do not grow with $n$; this ensures that each weight we store in our algorithms takes up $O(1)$ storage.
} 
where we obtain approximate MWM and $f$-MSM algorithms for matchings (a.k.a. hypermatchings) given a bound $p$ on the size of hyperedges. The second applies to maximization over the intersection of $p$ matroids (for a constant $p$ ): the case $p=2$ captures matchings in bipartite graphs. In each family, we have a maximum-submodular problem (MSIS, say - the "IS" stands for "independent set"), and a maximum-weight problem (MWIS, say) where the submodular function is modular. The results are summarized below; details appear in Sections 5 and 6 .

Theorem 3. For every submodular $f$, the MWIS and $f$-MSIS problems, with independent sets being given either by a hypermatching constraint in p-hypergraphs or by the intersection of $p$ matroids, there are nearlinear-space streaming algorithms giving the following approximation ratios.

\begin{tabular}{l|c|c} 
Problem type & MWIS & MSIS \\
\hline One pass: $p$-hypergraphs; $p$ matroids & $2(p+\sqrt{p(p-1)})-1$ & $4 p$ \\
$O\left(\varepsilon^{-3} \log p\right)$ passes: $p$-hypergraphs; p partition matroids & $p+\varepsilon$ & $p+1+\varepsilon$
\end{tabular}

The one-pass MWIS result for matroids was already known from the work of Ashwinkumar [2]; the remaining results in Theorem 3 are novel.

Our results for $f$-MSM and $f$-MSIS as stated above involve approximation ratios that are always worse than the corresponding ones for MWM and MWIS: therefore they are not generalizations in the strictest sense. However, such a generalization is possible by considering the curvature $\operatorname{curv}(f)$ of the submodular function $f: 2^{E} \rightarrow \mathbb{R}$. This quantity, defined by

$$
\operatorname{curv}(f):=\min \{c: \forall A \subseteq E \forall e \in E \backslash A \text {, we have } f(A \cup\{e\})-f(A) \geqslant(1-c) f(\{e\})\},
$$

measures how far $f$ is from being modular. Note that $\operatorname{curv}(f) \in[0,1]$ and $\operatorname{curv}(f)=0$ iff $f$ is modular. For our final result, we give approximation ratios for $f$-MSM and $f$-MSIS that gradually improve to those for Zelke's MWM algorithm and Ashwinkumar's MWIS algorithm as curv $(f) \rightarrow$ 0 .

Theorem 4. For every submodular $f$, the approximation ratios for $f$-MSM in Theorem 1 and the one-pass approximation ratio for $f$-MSIS in Theorem 3 can be improved to $\min \{7.75,5.585 /(1-\operatorname{curv}(f))\}$ and $\min \{4 p,(2(p+\sqrt{p(p-1)})-1) /(1-\operatorname{curv}(f))\}$ respectively.

\subsection{Context}

To place Theorems 1 and 2 in context, we summarize the most relevant prior work on MWM and MCM. As noted before, our work is the first to consider MSM.

A one-pass semi-streaming 2-approximation for MCM is trivial, from the observation that a maximal matching is a 2-approximate MCM. Beating this bound or proving its optimality remains a vexing open problem. Goel, Kapralov, and Khanna [11] showed that for every $\varepsilon>0$, finding a $(3 / 2-\varepsilon)$-approximate MCM in one pass requires $n^{1+\Omega(1 / \log \log n)}$ space, and very recently Kapralov [13] improved this to the best known approximation ratio lower bound of $\mathrm{e} /(\mathrm{e}-1) \approx$ 1.582. Using multiple passes, McGregor [18] did obtain a $(1+\varepsilon)$-approximation algorithm, but required $\exp \left(\varepsilon^{-1}\right)$ passes to do so.

For the MWM problem, Feigenbaum et al. [7] gave a one-pass semi-streaming 6-approximation algorithm, which McGregor [18] improved to $3+\sqrt{8} \approx 5.828$ by tweaking parameters. McGregor also extended his algorithm to multiple passes, where each pass essentially "repeats" the first pass, gradually improving the approximation ratio to $2+\varepsilon$ after $O\left(\varepsilon^{-3}\right)$ passes. Zelke further improved the one-pass approximation factor to $\approx 5.585$ (the exact constant is a degree- 5 algebraic 
number) by using a more involved algorithm. We shall have reason to discuss Zelke's and McGregor's algorithms in detail in Sections 3 and 4 respectively. Most recently, Epstein et al. [6] gave the current best approximation ratio of $\approx 4.911+\varepsilon$, using $O(n \log (n / \varepsilon))$ space. Their algorithm departs significantly from previous approaches, and falls outside our aforementioned framework. Very recently, Ahn and Guha [1] gave a $(1+\varepsilon)$ approximation with multiple passes, using a very different algorithm that falls outside our framework.

It is also worth noting another line of work on MWM that has focused on bipartite graphs, where it is natural to consider an alternate streaming model in which vertices arrive together with all their incident edges. The seminal online (randomized) algorithm of Karp, Vazirani, and Vazirani [14] falls in this setting and gives an e/ (e - 1) competitive ratio. Recent work of Goel, Kapralov, and Khanna [11] gave a deterministic semi-streaming algorithm with the same approximation ratio. Kapralov's aforementioned lower bound [13], which holds despite the bipartite and vertex-arrival restrictions, shows that this ratio is optimal.

\subsection{Other Related Work}

Thus far, we have been thinking about MSM as a problem about finding a good matching, generalizing MWM. Another useful viewpoint is to consider $f$-MSM as the problem of maximizing the submodular function $f(S)$ subject to $S$ being a matching. This makes MSM an instance of constrained submodular maximization, which is a heavily-studied topic in optimization.

Maximizing a submodular function is an important problem even without constraints on the set if one drops the monotonicity requirement. Even its most famous instance, MAX-CUT, is not yet fully understood. For monotone functions, maximizing $f(S)$ becomes nontrivial once one places some sort of "packing" constraint on $S$, such as an upper bound on $|S|$. Generalizing this idea naturally leads one to a matroid constraint, where $S$ is required to be an independent set of a matroid. One can consider more general "independence systems," such as the intersection of $p$ different matroids on the same ground set $E$ (called a $p$-intersection system) or, even more generally, a $p$-system, wherein

$$
\forall A \subseteq E: \frac{\max \{|S|: S \subseteq A, S \text { maximally independent }\}}{\min \{|S|: S \subseteq A, S \text { maximally independent }\}} \leqslant p .
$$

This last generalization finally captures the constraint of $S$ being a matching, because matchings form a 2-system. All of these classes of problems were studied in the seminal work of Fisher, Nemhauser and Wolsey [9, 20], who showed among other things that the simple greedy strategy of growing a set $S$ by adding the element that most improves $f(S)$ subject to $S$ being independent yields a $(p+1)$-approximation for a $p$-system. In another classical work, Jenkyns [12] showed that the greedy strategy with a $p$-system constraint in fact gives a $p$-approximate solution if the function $f$ is modular (a.k.a. linear).

Notice that the implication of the above for our $f$-MSM problem is a (non-streaming) greedy 3-approximation. This should be compared to our Theorem 2 .

More recently, Calinescu et al. [4] gave a polynomial time (e/ $(\mathrm{e}-1))$-approximation algorithm for maximizing a non-negative monotone proper submodular function $f$ subject to a matroid constraint. This ratio improves upon the 2 that follows from a matroid being a 1-system, and is provably optimal if $\mathrm{P} \neq \mathrm{NP}$. Lee et al. [15, 16] gave local search algorithms for maximizing $f$ over a $p$-intersection system, improving the approximation ratio from the aforementioned $p+1$ (for the more general $p$-systems) to $p+\varepsilon$. Recently, Feldman et al. [8] proposed a new class of independence systems called " $p$-exchange systems," stricter than $p$-systems but more general than $p$ intersection systems, for which they gave a local-search-based $(p+\varepsilon)$-approximation. Their paper 
is highly recommended for a concise yet comprehensive summary of relevant work on submodular maximization. Matchings form a 2-exchange system. Therefore this last result improved-after a span of over 30 years - the best known approximation ratio for $f$-MSM from 3 [9] to $2+\varepsilon$. We invite the reader to compare again with our Theorem 2 .

\subsection{Motivation and Significance of Our Results}

In applications such as big data analytics, it is sometimes preferable to compute a good solution quickly, even if a theoretically stronger guarantee can be achieved by a slower algorithm. Our algorithms in this work should be seen in this light: they are significant because they are faster algorithms with slightly worse approximation ratios than best known offline approximation algorithms. Moreover, they are able to handle input presented in streaming fashion, a clear advantage when handling big data.

Notably, none of the algorithmic strategies discussed in Section 1.3 are suitable for use with a graph stream. Let us focus just on our problem, $f$-MSM. For an $m$-edge graph, a typical step of the greedy strategy requires an examination of $\Omega(m)$ edges and $\Omega(m)$ calls to the $f$-oracle. The local search algorithms need to find a "good" local move in each step and in general it is not clear how a single pass over a graph stream can guarantee more than one such good move. So a local search algorithm that makes $\tau$ moves potentially translates into a $\tau$-pass streaming algorithm, and the best upper bound for $\tau$ in the aforementioned $(2+\varepsilon)$-approximation algorithm appears to be $O\left(\varepsilon^{-1} n^{4}\right)$. In contrast, our algorithms make $O(1)$ calls to the $f$-oracle per input item per pass, use a constant number of passes, and use an essentially optimal amount of storage.

Very recently, and concurrent with our work, Ashwinkumar and Vondrák [3] gave a $(p+1+$ $\varepsilon)$-approximation algorithm for submodular maximization over a $p$-system. Their algorithm can be thought of as using $O\left(\varepsilon^{-2} \log ^{2}(m / \varepsilon)\right)$ passes, where $m=|E|$. Our result in Theorem 3 uses fewer passes (for constant $\varepsilon$ ), but handles a smaller class of constraints than $p$-systems.

As noted at the start, the generalization from MWM to MSM has some practical motivation, such as for the WAP problem from computational linguistics [17]. In addition, we feel that the MSM problem is a pleasing marriage of submodular maximization, data streaming, and matching theory; and more generally, MSIS brings together submodularity, streaming, and matroids.

\section{Preliminaries}

We start by making our model of computation precise. The input is an $n$-vertex graph stream, defined as a sequence $\sigma=\left\langle e_{1}, e_{2}, \ldots, e_{m}\right\rangle$ of distinct edges, where each $e_{i}=\left(u_{i}, v_{i}\right) \in[n] \times[n]$ and $u_{i}<v_{i}$. We put $V=[n], E=\left\{e_{1}, \ldots, e_{m}\right\}$, and $G=(V, E)$. The submodular function $f: 2^{E} \rightarrow \mathbb{R}_{+}$, which is part of the problem specification, is given by an entity external to the stream, called the value oracle for $f$, or the $f$-oracle. A data stream algorithm, after reading each edge from the input stream, is allowed to make an arbitrary number of calls to the $f$-oracle. (In fact the algorithms we design here make only $O(1)$ such calls on average.) A call consists of the algorithm sending the oracle a subset $S \subseteq E$, whereupon the oracle returns the value $f(S)$ in constant time. The space required to describe $S$ counts towards the algorithm's space usage.

Notice that $f$ is only defined on subsets of $E$, and the most important restriction on the algorithm is that at any time it can only remember a tiny portion of $E$. To prevent the algorithm from "cheating" and learning about $E$ indirectly from oracle calls, we say that the algorithm fails or aborts if it ever tries to obtain $f(S)$ with $S \nsubseteq E$. 


\subsection{Hardness of MSM}

We cannot hope to solve MSM exactly. As noted in Section 1.2, even the very special case MCM cannot be approximated any better than e/ $(\mathrm{e}-1)$ in the semi-streaming setting [13]. But the data stream model does not adequately capture the vast gulf between MWM and MSM.

Theorem 5. For every $C<\mathrm{e} /(\mathrm{e}-1)$, there does not exist a polynomial-time $C$-approximation algorithm for $f$-MSM relative to a value oracle for $f$.

Proof. Given a submodular $f$, we can view $f$-MSM as a generalization of the constrained maximization problem $\max _{|S| \leqslant k} f(S)$ : consider $f$-MSM on a disjoint union of $k$ star graphs. Nemhauser and Wolsey [19, Theorem 4.2] show that for the latter problem, given the upper bound on $C$, a $C$ approximation algorithm must make a superpolynomial number of calls to the $f$-oracle.

\subsection{A Framework for Streaming MSM and MSIS Algorithms}

We proceed to describe a generic streaming algorithm for $f$-MSM, which defines the framework alluded to in Section 1.1. In fact, as noted towards the end of Section 1.1, our framework applies to the much more general problem of $f$-MSIS (Maximum Submodular Independent Set), an instance of which is given by a submodular $f: 2^{E} \rightarrow \mathbb{R}_{+}$and a collection $\mathcal{I} \subseteq 2^{E}$ of independent sets such that $\varnothing \in \mathcal{I}$. We put $m:=|E|$ and $n:=\max _{I \in \mathcal{I}}|I|$. We assume that independence (i.e., membership in $\mathcal{I}$ ) can be tested easily; we require no other structural property of $\mathcal{I}$. For the special case of MSM, $\mathcal{I}$ is the collection of matchings in a graph with edge set $E$.

The generic algorithm for $f$-MSIS starts with a given independent set $P$ (possibly empty) and then proceeds to make one pass over the input stream $\sigma$, attempting to end up with an improved independent set $I$ by the end of the pass. The algorithm processes the elements in $E$ in a pretend stream order that consists of an arbitrary permutation of the elements in $P$, followed by the elements in $E \backslash P$ in the same order as $\sigma$. Throughout, the algorithm maintains a "current solution" $I \in \mathcal{I}$, a set $S \subseteq E$ of "shadow elements" (this term is borrowed from Zelke [21]), and a weight $w(e)$ for each element $e$ it has processed. The algorithm bases its decisions on a real-valued parameter $\gamma>0$. For a set $A \subseteq E$, we denote $w(A):=\sum_{e \in A} w(e)$. An augmenting pair for a set $I \in \mathcal{I}$ is a pair of sets $(A, J)$ such that $J \subseteq I$ and $(I \backslash J) \cup A \in \mathcal{I}$. For $e \in E$, define $A+e$ to be $A \cup\{e\}$.

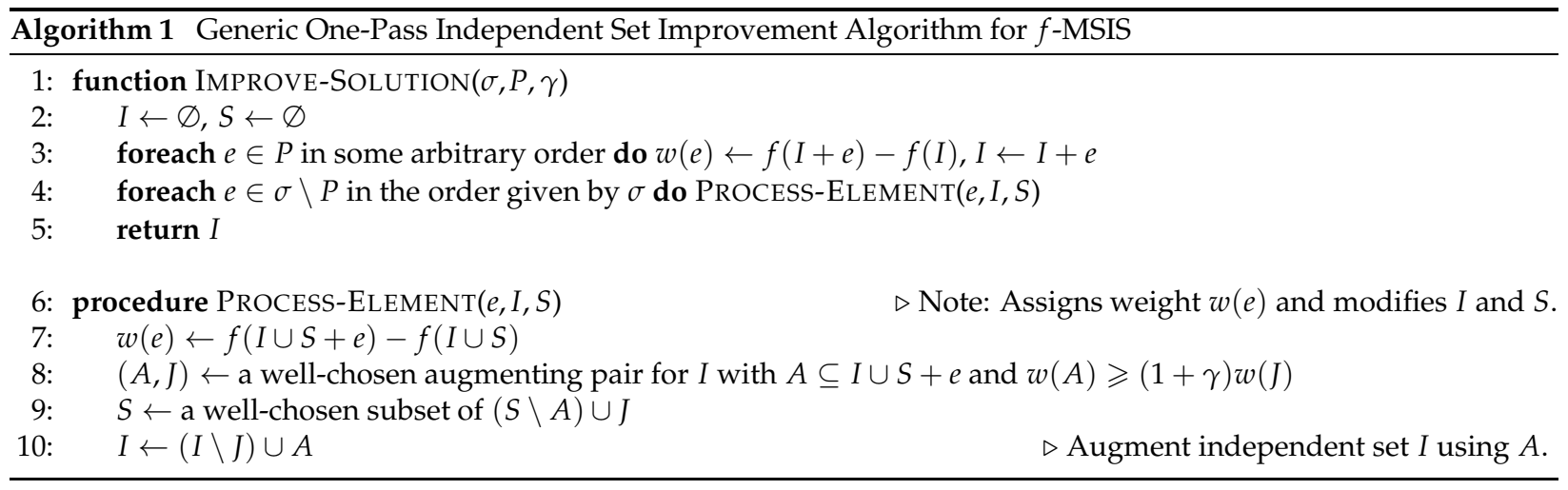

Notice that PROCESS-ELEMENT maintains the invariant that $w(e)$ is defined for all $e \in I \cup S$. Therefore, Line 8 never tries to access an element weight before defining it. Furthermore, the algorithm need only remember the weights of elements in $I \cup S$. Therefore, the space usage of the algorithm is bounded by $O((|P|+|I|+|S|) \log m)=O((n+|S|) \log m)$, since $P$ and $I$ are independent sets. 
To instantiate this generic algorithm, one must specify the precise logic used in Lines 8 and 9 . If the algorithm is for MWIS rather than MSIS, then $w(e)$ values are already given and assignments to those values (see Lines 3 and 7 ) should be ignored.

Definition 6. We say that an MWIS algorithm is compliant if each pass instantiates Algorithm 1 in the above sense, i.e., it starts with some solution $P \in \mathcal{I}$ computed in the previous pass and calls $\operatorname{ImPROVE-SOLUTION}(\sigma, P, \gamma)$. The parameter $\gamma$ need not be the same for all passes.

Definition 7. For a submodular $f$, we define an $f$-extension of a compliant MWIS algorithm $\mathcal{A}$ to be Algorithm 1 , with the logic used in Lines 8 to 9 being borrowed from $\mathcal{A}$, and with values of the parameter $\gamma$ possibly differing from those used by $\mathcal{A}$.

Lemma 8 (Modular to submodular). Let $\mathcal{A}$ be a one-pass compliant MWIS algorithm that computes a $C_{\gamma}$-approximate MWIS when run with parameter $\gamma$. Then, for every non-negative monotone proper submodular $f$, its $f$-extension with parameter $\gamma$ computes $a\left(C_{\gamma}+1+1 / \gamma\right)$-approximate $f$-MSIS.

The rest of the paper is organized as follows. Section 3 develops some important properties of compliant algorithms and proves Lemma 8. Applying this lemma, the $f$-extension of Zelke's one-pass MWM algorithm [21] is easily shown to yield a 7.75-approximation for $f$-MSM, proving Theorem 1. Section 4 revisits McGregor's multi-pass algorithm and analysis [18], extends his analysis, and obtains a $(3+\varepsilon)$-approximation for $f$-MSM, proving Theorem 2 . Sections 5 and 6 briefly discuss our results for MWM and MSM in hypergraphs, and MWIS and MSIS for the intersection of $p$ matroids. Technical details of these results are given in the appendix.

\section{A One-Pass Solution via Compliant Algorithms}

Consider the $f$-extension with parameter $\gamma$ of a particular one-pass compliant algorithm. Let $I$ denote its output, i.e., the result of invoking IMPROVE-SOLUTION $(\sigma, \varnothing, \gamma)$ and $I^{*}$ be an $f$-MSIS. Let $I_{e}, S_{e}$ denote the contents of the variables $I, S$ in Algorithm 1 just before element $e$ is processed. Let $K=\left(\bigcup_{e \in E} I_{e}\right) \backslash I$ denote the set of elements that were added to the current solution at some point but were killed and did not make it to the final output. Then $\bigcup_{e \in E} S_{e} \subseteq I \cup K$, because an element can become a shadow element only when it was removed from the current solution at some point (see Line 9 of Algorithm 1 ). Hence

$$
\bigcup_{e \in E}\left(I_{e} \cup S_{e}\right) \subseteq I \cup K
$$

Lemma 9. For an $f$-extension of a compliant algorithm, we have $w(K) \leqslant w(I) / \gamma$.

Proof. Let $A_{e}, J_{e}$ be the sets $A, J$ chosen at Line 8 when processing $e$. Each augmentation by $A_{e}$ (Line 10) increases the weight of the current solution by $w\left(A_{e}\right)-w\left(J_{e}\right) \geqslant \gamma w\left(J_{e}\right)$. Hence, $w(I) / \gamma \geqslant \sum_{e \in E} w\left(J_{e}\right)$.

The set $\bigcup_{e \in E} J_{e}$ consists of elements that were removed from the current solution at some point. Thus, it includes $K$ (the inclusion may be proper: $K$ does not contain elements that were removed from the current solution, reinserted, and eventually ended up in I). Therefore,

$$
w(K) \leqslant w\left(\bigcup_{e \in E} J_{e}\right) \leqslant \sum_{e \in E} w\left(J_{e}\right) \leqslant w(I) / \gamma .
$$

Lemma 10. For an f-extension of a compliant algorithm, we have $w(I) \leqslant f(I)$. 
Proof. Let $e_{1}^{I}, e_{2}^{I}, \ldots, e_{s}^{I}$ be an enumeration of $I$ in order of processing, where $s=|I|$. The logic of Algorithm 1 ensures that an element once removed from the shadow set can never return to the current solution (though elements can move between the two arbitrarily). Thus, $I \cap\left(I_{e_{i}^{I}} \cup S_{e_{i}^{I}}\right)=$ $\left\{e_{1}^{I}, e_{2}^{I}, \ldots, e_{i-1}^{I}\right\}$. Since $I \cap\left(I_{e_{i}^{I}} \cup S_{e_{i}^{I}}\right) \subseteq\left(I_{e_{i}^{I}} \cup S_{e_{i}^{I}}\right)$ and $f$ is submodular, Equation (1) gives

$$
f\left(\left\{e_{1}^{I}, e_{2}^{I}, \ldots, e_{i}^{I}\right\}\right)-f\left(\left\{e_{1}^{I}, e_{2}^{I}, \ldots, e_{i-1}^{I}\right\}\right) \geqslant f\left(I_{e_{i}^{I}} \cup S_{e_{i}^{I}}+e_{i}^{I}\right)-f\left(I_{e_{i}^{I}} \cup S_{e_{i}^{I}}\right)=w\left(e_{i}^{I}\right) .
$$

Summing this over $i \in[s]$ gives $f(I)=f(I)-f(\varnothing) \geqslant \sum_{i=1}^{s} w\left(e_{i}^{I}\right)=w(I)$.

Lemma 11. For an $f$-extension of a compliant algorithm, we have $f\left(I^{*}\right) \leqslant(1 / \gamma+1) f(I)+w\left(I^{*}\right)$.

Proof. Let $e_{1}^{B}, \ldots, e_{b}^{B}$ be an enumeration of $B:=I \cup K$ in order of processing. The set $B_{i}:=$ $\left\{e_{1}^{B}, \ldots, e_{i-1}^{B}\right\}$ consists of elements inserted into the current solution before $e_{i}^{B}$ was processed. Meanwhile $I_{e_{i}^{B}} \cup S_{e_{i}^{B}}$ is the subset of these elements that were not removed before $e_{i}^{B}$ was processed. Thus, $B_{i} \supseteq I_{e_{i}^{B}} \cup S_{e_{i}^{B}}$ for all $i \in[b]$. By submodularity of $f$ and Equation (1),

$$
f\left(\left\{e_{1}^{B}, e_{2}^{B}, \ldots, e_{i}^{B}\right\}\right)-f\left(\left\{e_{1}^{B}, e_{2}^{B}, \ldots, e_{i-1}^{B}\right\}\right) \leqslant f\left(I_{e_{i}^{B}} \cup S_{e_{i}^{B}}+e_{i}^{B}\right)-f\left(I_{e_{i}^{B}} \cup S_{e_{i}^{B}}\right)=w\left(e_{i}^{B}\right) .
$$

Summing this over $i \in[b]$ gives $f(B)=f(B)-f(\varnothing) \leqslant w(B)$. Thus, we have

$$
f(I \cup K) \leqslant w(I \cup K)=w(I)+w(K) \leqslant f(I)+w(I) / \gamma=(1 / \gamma+1) f(I),
$$

where the last two inequalities use Lemma 10 and Lemma 9 respectively.

Now we bound $f\left(I^{*}\right)$. Let $I^{*} \backslash(I \cup K)=\left\{e_{1}^{I^{*}}, e_{2}^{I^{*}}, \ldots, e_{t}^{I^{*}}\right\}$; this enumeration is in arbitrary order. Put $D_{0}=I \cup K, D_{i}=I \cup K \cup\left\{e_{1}^{I^{*}}, \ldots, e_{i}^{I^{*}}\right\}$ for $i \in[t]$. By Equation (3)), $D_{i-1} \supseteq I \cup K \supseteq$ $I_{e_{i}^{I^{*}}} \cup S_{e_{i}^{I^{*}}}$. Appealing to submodularity and Equation (1) again,

$$
f\left(D_{i}\right)-f\left(D_{i-1}\right) \leqslant f\left(I_{e_{i}^{I^{*}}} \cup S_{e_{i}^{I^{*}}}+e_{i}^{I^{*}}\right)-f\left(I_{e_{i}^{I^{*}}} \cup S_{e_{i}^{I^{*}}}\right)=w\left(e_{i}^{I^{*}}\right) .
$$

Summing this over $i \in[t]$ gives $f\left(D_{t}\right)-f\left(D_{0}\right) \leqslant w\left(I^{*} \backslash(I \cup K)\right) \leqslant w\left(I^{*}\right)$. In other words, $f\left(I \cup K \cup I^{*}\right)-f(I \cup K) \leqslant w\left(I^{*}\right)$. By monotonicity of $f$ and Equation (4) , we have

$$
f\left(I^{*}\right) \leqslant f\left(I \cup K \cup I^{*}\right) \leqslant f(I \cup K)+w\left(I^{*}\right) \leqslant(1 / \gamma+1) f(I)+w\left(I^{*}\right) .
$$

Proof of Lemma 8 . Since the compliant algorithm $\mathcal{A}$ outputs a $C_{\gamma}$-approximate MWIS, it satisfies $w\left(I^{*}\right) \leqslant C_{\gamma} w(I)$ for any weight assignment; in particular, the weights assigned by its $f$-extension. Using Lemma 11 and Lemma 10 , we conclude that $f\left(I^{*}\right) \leqslant\left(C_{\gamma}+1+1 / \gamma\right) f(I)$.

Proof of Theorem 1. Recall that an "independent set" is just a matching in the setting of MWM and MSM. Zelke's algorithm chooses the augmenting pair $(A, J)$ as follows: $A$ is chosen from an $O(1)$-sized "neighborhood" of the edge $e$ being processed, and $J$ is set to be $M\ulcorner A$ : the set of edges in $M$ that share a vertex with some edge in $A$. It chooses $S$ so that each shadow edge intersects some edge in the current matching, thus enforcing $|S|=O(n)$ and a space bound of $O(n \log n)$ bits. For the reader's convenience we spell out the logic of the algorithm in full in Appendix C.

Zelke's algorithm is compliant with $C_{\gamma}=2(1+\gamma)+(1 / \gamma+1)-\gamma /(1+\gamma)^{2}$ [21, Theorem 3]. By Lemma 8 , its $f$-extension yields an approximation ratio of $2(1+\gamma)^{2} / \gamma-\gamma /(1+\gamma)^{2}$, which attains a minimum value of 7.75 at $\gamma=1$. This proves the theorem. 


\subsection{Approximation Ratio in Terms of Curvature}

We now show how to obtain the stronger guarantee for $f$-MSM given in Theorem 4 . The tool we need is the following strengthening of Lemma 8 .

Lemma 12. Let $\mathcal{A}$ be a one-pass compliant MWIS algorithm that computes a $C_{\gamma}$-approximate MWIS when run with parameter $\gamma$. Then, for every non-negative monotone proper submodular $f$, its $f$-extension with parameter $\gamma$ computes a $\min \left\{C_{\gamma}+1+1 / \gamma, C_{\gamma} /(1-\operatorname{curv}(f))\right\}$-approximate $f$-MSIS.

Proof. We can bound $f\left(I^{*}\right)$ as follows.

$$
\begin{array}{rlrl}
f\left(I^{*}\right) & \leqslant \sum_{e \in I^{*}} f(\{e\}) & & \text { by submodularity of } f \\
& \leqslant \frac{1}{1-\operatorname{curv}(f)} \sum_{e \in I^{*}}\left(f\left(I_{e} \cup S_{e}+e\right)-f\left(I_{e} \cup S_{e}\right)\right) & & \text { by definition of curvature } \\
& =\frac{w\left(I^{*}\right)}{1-\operatorname{curv}(f)} & \\
& \leqslant \frac{w(I) C_{\gamma}}{1-\operatorname{curv}(f)} & & \text { by } C_{\gamma} \text {-approximation guarantee of } \mathcal{A} \\
& \leqslant \frac{f(I) C_{\gamma}}{1-\operatorname{curv}(f)} & & \text { by Lemma } 10 .
\end{array}
$$

Thus the $f$-extension of $\mathcal{A}$ achieves an approximation ratio of at most $C_{\gamma} /(1-\operatorname{curv}(f))$. Combining this with Lemma 8 completes the proof.

Proof of Theorem 4 . We appeal to Lemma 12. Recall the expression for $C_{\gamma}$ for Zelke's algorithm, given in the proof of Theorem 1 above. For $f$-MSM, the claimed approximation ratio follows by picking the better of the two solutions obtained by running two $f$-extensions of Zelke's algorithm in parallel: one with $\gamma=0.717$, which minimizes $C_{\gamma}$, and another with $\gamma=1$, which minimizes $C_{\gamma}+1+1 / \gamma$.

A similar idea applied to the appropriate compliant algorithms (outlined in Sections 5 and 6 ) gives the claimed results for $f$-MSIS on $p$-hypergraphs and $p$-intersection systems.

Note that, in all of these cases, we can avoid having to run two parallel $f$-extensions if we knew $\operatorname{curv}(f)$ in advance, for we could then simply figure out which value of $\gamma$ gives the better approximation ratio. For instance, in the case of $f$-MSM, we would pick $\gamma=1$ if 5.585/(1$\operatorname{curv}(f)) \geqslant 7.75$ and $\gamma=0.717$ otherwise.

\section{A Multi-Pass MSM Algorithm}

In this section we prove Theorem 2. For this we first review McGregor's multi-pass MWM algorithm [18], which is compliant. Our algorithm is simply its $f$-extension, as explained in Section 2.2.

To describe McGregor's algorithm with respect to our framework (Algorithm1), we need only explain the two choices made inside PROCESS-EDGE. These are especially simple. The algorithm never creates any shadow edges, so Line 9 always chooses $S=\varnothing$. In Line 8 , the augmenting pair $(A, J)$ is chosen so that $A=\{e\}$ if possible, and $A=\varnothing$ otherwise, and $J=M \Gamma A$. Recall that $M\ulcorner A$ denotes the set of edges in matching $M$ that share a vertex with some edge in set $A$. This describes a single pass. The overall algorithm starts with an empty matching and repeatedly invokes IMPROVE-MATCHING with $\gamma=1 / \sqrt{2}$ for the first pass and $\gamma=2 \varepsilon / 3$ for the remaining 
passes. It stops when the multiplicative improvement made in a pass drops below a certain wellchosen rational function of $\gamma$. McGregor analyzes this algorithm to show that it makes at most $O\left(\varepsilon^{-3}\right)$ passes and terminates with a $(2+2 \varepsilon)$-approximate MWM.

In our $f$-extension, we make the following tweaks to the parameter $\gamma$ : we use $\gamma=1$ for the first pass and $\gamma=\varepsilon / 3$ for the remaining passes. For the reader's convenience, we lay out the logic of the resulting $f$-MSM algorithm explicitly in Algorithm 2. The function IMPROVE-MATCHING is exactly as in Algorithm 1 except that it calls Process-EdGE $(e, M)$, since $S$ is never used.

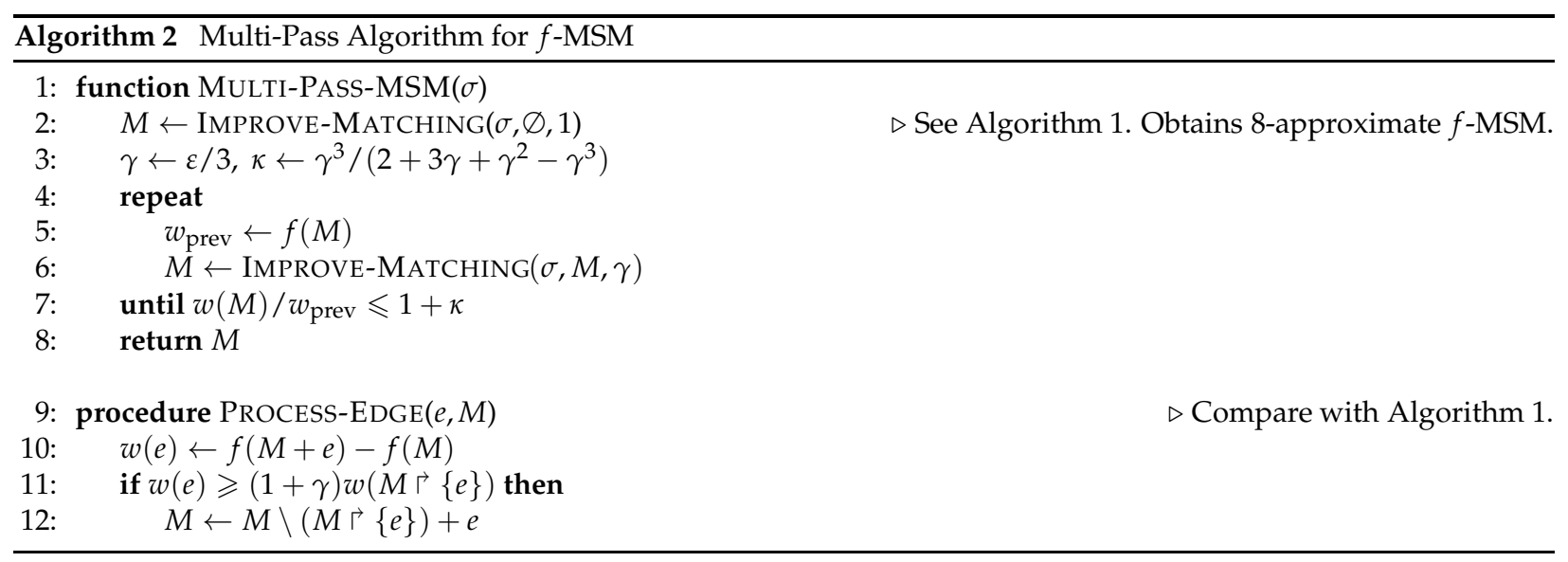

Let $M^{i}$ denote the matching $M$ computed by Algorithm 2 at the end of its $i$ th pass over $\sigma$. When an edge $e$ is added to $M$ in Line 12, we say that $e$ is born and that it kills the (at most two) edges in $M \Gamma\{e\}$. Notice that during pass $i>1$, thanks to the pretend stream order in which edges are processed, initially all edges in $M^{i-1}$ are born without killing anybody2 (cf. the discussion at the start of Section 2.2); for the rest of the pass these edges are never considered for addition to $M$.

Let $K^{i}$ denote the set of edges killed during pass $i$ (some of them may be born during a subsequent pass). Then $M^{i} \cup K^{i}$ is exactly the set of edges born in pass $i$. These edges can be made the nodes of a collection of disjoint rooted killing trees 3 where the parent of a killed edge $e$ is the edge $e^{\prime}$ that killed it. The set of roots of these killing trees is precisely $M^{i}$. Let $T^{i}(e)$ denote the set of strict descendants of $e \in M^{i}$ in its killing tree. Then $K^{i}=\bigcup_{e \in M^{i}} T^{i}(e)$.

Let $B^{i}=M^{i} \cap M^{i-1}$ denote the set of edges that pass $i$ retains in the matching from the previous pass. By the preceding discussion, it follows that $T^{i}(e)=\varnothing$ for all $e \in B^{i}$.

\subsection{Analysis}

We now analyze Algorithm 2. As before, let $M^{*}$ denote an optimal solution to the $f$-MSM instance. We first prove an approximation guarantee for the first pass. It is not the best possible one-pass result (see Theorem 11), but an $O(1)$-approximation suffices, so we can use the simpler algorithm.

Lemma 13. The matching $M^{1}$ is an 8-approximate $f$-MSM, i.e., $f\left(M^{1}\right) \geqslant f\left(M^{*}\right) / 8$.

Proof. The first pass of the algorithm is a one-pass compliant algorithm. As shown by McGregor [18, Lemma 3], its approximation factor is $C_{\gamma}=1 / \gamma+3+2 \gamma$. Applying Lemma 8, we have $f\left(M^{*}\right) / f\left(M^{1}\right) \leqslant 2 / \gamma+4+2 \gamma$. This bound is minimized at $\gamma=1$ (explaining the choice made in Line 2) at which point it evaluates to 8 .

\footnotetext{
${ }^{2}$ This subtlety appears to have been missed in McGregor's analysis [18] and it creates a gap in his argument. Using a pretend stream order as we do in this work fixes that gap.

${ }^{3}$ Feigenbaum et al. [7] and McGregor [18] used the evocative term "trail of the dead" for this concept.
} 
Define $\tau$ to be the number of passes made by Algorithm 2. Let $w_{i}(e)$ denote the weight assigned to edge $e$ in Line 10 during the $i$ th pass. For the rest of this section, $\gamma$ denotes the parameter value used by passes 2 through $\tau$, and $\kappa$ denotes the corresponding value assigned at Line 3. To analyze the result of those passes, we first borrow three results-stated in the next three lemmas-from McGregor's analysis [18, Lemma 3 and Theorem 3], which in turn borrows from the Feigenbaum et al. analysis [7, Theorem 2].

Lemma 14. For all $i \in[2, \tau]$ and all $e \in M^{i}$, we have $w_{i}\left(T^{i}(e)\right) \leqslant w_{i}(e) / \gamma$.

Proof. Directly analogous to Lemma 9 .

Lemma 15. We have $w_{\tau}\left(B^{\tau}\right) / w_{\tau}\left(M^{\tau}\right) \geqslant(\gamma-\kappa) /(\gamma+\gamma \kappa)$.

Proof sketch. The logic in Lines 11 to 12 ensures that, for all $i \in[2, \tau]$, we have $w_{i}\left(M^{i} \backslash B^{i}\right) \geqslant$ $(1+\gamma) w_{i}\left(M^{i-1} \backslash B^{i}\right)$. In particular, this inequality holds at $i=\tau$.

During the initial phase of pass $i \geqslant 2$, the set $M$ is monotonically built up from $\varnothing$ to $M^{i-1}$ according to a pretend stream order and weights are assigned to edges in $M^{i-1}$ according to Line 10 . Because of this monotonicity, summing the weights of these edges causes the $f$ terms to telescope, giving $w_{i}\left(M^{i-1}\right)=f\left(M^{i-1}\right)$. So the stopping criterion in Line 7 ensures that $w_{\tau}\left(M^{\tau}\right) / w_{\tau}\left(M^{\tau-1}\right) \leqslant$ $1+\kappa$. Combining this with the inequality in the last paragraph (at $i=\tau$ ) yields the lemma after some straightforward algebra.

Lemma 16. For all $i \in[2, \tau]$, we have $w_{i}\left(M^{*}\right) \leqslant(1+\gamma) \sum_{e \in M^{i}}\left(w_{i}\left(T^{i}(e)\right)+2 w_{i}(e)\right)$.

Proof sketch. This lemma has a rather creative proof, wherein the weights of edges in $M^{*}$ are charged to edges in $M^{i} \cup K^{i}$ using a careful charge transfer scheme. For the sake of completeness we give a full proof in Appendix $\mathrm{A}$.

We are now ready to fully analyze the approximation guarantee and complexity of Algorithm 2, thereby proving Theorem 2 .

Proof of Theorem 2. As noted earlier, $T^{i}(e)=\varnothing$ for all $e \in B^{i}$ and $K^{i}=\bigcup_{e \in M^{i}} T^{i}(e)$. Therefore, $K^{\tau}=\bigcup_{e \in M^{\tau} \backslash B^{\tau}} T^{\tau}(e)$, which gives

$$
w_{\tau}\left(K^{\tau}\right)=\sum_{e \in M^{\tau} \backslash B^{\tau}} w_{\tau}\left(T^{\tau}(e)\right) \leqslant \sum_{e \in M^{\tau} \backslash B^{\tau}} \frac{w_{\tau}(e)}{\gamma}=\frac{w_{\tau}\left(M^{\tau} \backslash B^{\tau}\right)}{\gamma}=\frac{w_{\tau}\left(M^{\tau}\right)-w_{\tau}\left(B^{\tau}\right)}{\gamma},
$$

where the inequality follows from Lemma 14. Using Equation (6) and relating the first and third terms in Equation (4), we get

$$
f\left(M^{\tau} \cup K^{\tau}\right) \leqslant w_{\tau}\left(M^{\tau}\right)+\frac{w_{\tau}\left(M^{\tau}\right)-w_{\tau}\left(B^{\tau}\right)}{\gamma}=\left(1+\frac{1}{\gamma}\right) w_{\tau}\left(M^{\tau}\right)-\frac{1}{\gamma} w_{\tau}\left(B^{\tau}\right) .
$$


Using Lemma16, we now get

$$
\begin{aligned}
w_{\tau}\left(M^{*}\right) & \leqslant(1+\gamma) \sum_{e \in M^{\tau}}\left(w_{\tau}\left(T^{\tau}(e)\right)+2 w_{\tau}(e)\right) \\
& =(1+\gamma)\left[\left(\sum_{e \in M^{\tau} \backslash B^{\tau}} w_{\tau}\left(T^{\tau}(e)\right)\right)+2 w_{\tau}\left(M^{\tau}\right)\right] \quad \text { since } \forall e \in B^{\tau} \text {, we have } T^{\tau}(e)=\varnothing \\
& \leqslant(1+\gamma)\left[\left(\sum_{e \in M^{\tau} \backslash B^{\tau}} \frac{w_{\tau}(e)}{\gamma}\right)+2 w_{\tau}\left(M^{\tau}\right)\right] \quad \text { using Lemma14 } \\
& =\frac{1+\gamma}{\gamma}\left(w_{\tau}\left(M^{\tau}\right)-w_{\tau}\left(B^{\tau}\right)\right)+(2+2 \gamma) w_{\tau}\left(M^{\tau}\right) \\
& =\left(\frac{1}{\gamma}+3+2 \gamma\right) w_{\tau}\left(M^{\tau}\right)-\left(1+\frac{1}{\gamma}\right) w_{\tau}\left(B^{\tau}\right) .
\end{aligned}
$$

By using Equation (5), we have $f\left(M^{*}\right) \leqslant f\left(M^{\tau} \cup K^{\tau}\right)+w_{\tau}\left(M^{*}\right)$. So using Equation (7) we get

$$
\begin{array}{rlrl}
f\left(M^{*}\right) & \leqslant\left(1+\frac{1}{\gamma}\right) w_{\tau}\left(M^{\tau}\right)-\frac{1}{\gamma} w_{\tau}\left(B^{\tau}\right)+\left(\frac{1}{\gamma}+3+2 \gamma\right) w_{\tau}\left(M^{\tau}\right)-\left(1+\frac{1}{\gamma}\right) w_{\tau}\left(B^{\tau}\right) \\
& =w_{\tau}\left(M^{\tau}\right)\left[1+\frac{1}{\gamma}+\frac{1}{\gamma}+3+2 \gamma\right]-w_{\tau}\left(B^{\tau}\right)\left[1+\frac{2}{\gamma}\right] \\
& \leqslant\left[4+\frac{2}{\gamma}+2 \gamma-\left(1+\frac{2}{\gamma}\right) \frac{\gamma-\kappa}{\gamma+\gamma \kappa}\right] w_{\tau}\left(M^{\tau}\right) & \text { using Lemma 15 } \\
& =(3+3 \gamma) w_{\tau}\left(M^{\tau}\right) & \text { substituting } \kappa=\frac{\gamma^{3}}{2+3 \gamma+\gamma^{2}-\gamma^{3}}, \\
& \leqslant(3+\varepsilon) f\left(M^{\tau}\right) & \text { using Lemma 10 }
\end{array}
$$

and this completes the proof that Algorithm 2 computes a $(3+\varepsilon)$-approximate $f$-MSM.

Finally we bound the number of passes made by the algorithm. This requires some care. It is not as immediate as the corresponding analysis in McGregor's MWM algorithm, because the weight function $w_{i}$ changes from pass to pass. We proceed as follows.

For all $i \in[2, \tau-1]$, we have

$$
f\left(M^{i}\right) \geqslant w_{i}\left(M^{i}\right)>(1+\kappa) f\left(M^{i-1}\right),
$$

where the first inequality uses Lemma 10, and the second uses the stopping criterion in Line 7 . Since $M^{*}$ is optimal, $f\left(M^{*}\right) \geqslant f\left(M^{i}\right)$, and repeated application of the above inequality gives us

$$
f\left(M^{*}\right) \geqslant f\left(M^{i}\right)>(1+\kappa)^{i-1} f\left(M^{1}\right) \geqslant(1+\kappa)^{i-1} f\left(M^{*}\right) / 8,
$$

where the final step uses Lemma 13. Applying this at $i=\tau-1$ gives $\tau \leqslant 2+\log _{1+\kappa} 8=O\left(\kappa^{-1}\right)=$ $O\left(\gamma^{-3}\right)=O\left(\varepsilon^{-3}\right)$. Thus, the algorithm finishes in $O\left(\varepsilon^{-3}\right)$ passes, as claimed.

\section{Generalization to Matchings in Hypergraphs}

A hypergraph is a pair $H=(V, E)$, where $V$ is a finite set and $E$ is a collection of subsets of $V$. It is a $p$-hypergraph if $|e| \leqslant p$ for all $e \in E$. A matching in $H$ is a pairwise disjoint subcollection of $E$. McGregor's one-pass and multi-pass algorithms for MWM and its $f$-extensions we gave above 
can be generalized to compute approximate MWMs and $f$-MSMs in $p$-hypergraphs. For MWM in $p$-hypergraphs, we get approximation ratios of $2(p+\sqrt{p(p-1)})-1$ in one pass and $p+\varepsilon$ with $O\left(\varepsilon^{-3} \log p\right)$ passes. For MSM, the respective approximation ratios we obtain are $4 p$ and $p+1+\varepsilon$. We define $n:=|V|$ and $m:=|E| \leqslant n^{p}$, so the space usage is $O(n \log n)$.

To obtain these results, we generalize the charging scheme alluded to in the proof of Lemma 16 . We argue that weights of edges in $M^{*}$ can be charged and these charges redistributed such that

$$
w\left(M^{*}\right) \leqslant \sum_{e \in M}(1+\gamma)((p-1) w(T(e))+p w(e))
$$

where $T(e)$ is the set of non-roots in the killing tree of $e$. This argument appears in Appendix A.

The rest of the analysis is identical in all four cases $\{\mathrm{MWM}, \mathrm{MSM}\} \times\{$ one-pass, multi-pass $\}$. In the multi-pass algorithms, we have to set values of $\gamma$ and $\kappa$. We use $\gamma=\varepsilon /(p+1)$ for both MSM and MWM. We use $\kappa=\gamma^{3} /\left((p-1)(1+\gamma)^{2}-\gamma^{3}\right)$ for MWM and $\kappa=\gamma^{3} /(p+(2 p-1) \gamma+$ $\left.(p-1) \gamma^{2}-\gamma^{3}\right)$ for MSM to get the desired approximation ratios for Theorem 3 .

To get better approximation ratios in terms of $\operatorname{curv}(f)$, as stated in Theorem 4 , we again appeal to Lemma 12, applying it to the compliant one-pass hypergraph MWM algorithm. We can then use the same idea as in Section 3 , taking the better of two values of $\gamma$, to obtain a one-pass approximation ratio of $\min \{4 p,(2(p+\sqrt{p(p-1)})-1) /(1-\operatorname{curv}(f))\}$.

\section{Maximization Over (Multiple) Matroids}

A matroid is a pair $M=(E, \mathcal{I})$ such that $E$ is a finite set, $\mathcal{I}$ is a collection of subsets of $E$, and the following conditions hold: (1) $\varnothing \in \mathcal{I}$; (2) if $I \in \mathcal{I}$ and $J \subseteq I$, then $J \in \mathcal{I}$ (in other words, $\mathcal{I}$ is closed under the subset operation); (3) if $I, J \in \mathcal{I}$ and $|J|<|I|$, then there exists an element $e \in I-J$ such that $J+e \in \mathcal{I}$. A set $I \in \mathcal{I}$ is also called an independent set. A maximally independent set is a base of the matroid. A set that is not independent is dependent. A minimally dependent set is a circuit. Since the number of independent sets in a matroid can be exponential, we assume that access to $\mathcal{I}$ is via an oracle. When a set $I$ is passed to this oracle, it returns an empty set if $I$ is independent, otherwise it returns a circuit in $I$.

Given $p$ matroids $M_{1}=\left(E, \mathcal{I}_{1}\right), \ldots, M_{p}=\left(E, \mathcal{I}_{p}\right)$ over the same ground set $E$, and a nonnegative monotone proper submodular function $f: 2^{E} \rightarrow \mathbb{R}_{+}$, we consider the problem of finding $\operatorname{argmax}_{I \in \mathcal{I}_{1} \cap \cdots \cap \mathcal{I}_{p}} f(I)$, in the streaming model, where elements of $E$ arrive in the stream. We define $m:=|E|$ and $n:=\max _{I \in \mathcal{I}_{1} \cap \cdots \cap \mathcal{I}_{p}}|I|$. Then our algorithms use $O\left(n(\log m)^{O(1)}\right)$ memory.

Ashwinkumar [2] gave a one-pass $(2(p+\sqrt{p(p-1)})-1)$-approximation algorithm (and a matching lower bound when the algorithm is only allowed to store a feasible solution) when $f$ is modular. His algorithm, which we explain in Appendix B, is compliant. Hence, by Lemma 8 , we get an approximation ratio of $4 p$ when $f$ is submodular (by setting $\gamma=1$ ). Now, we seek to improve this ratio using multiple passes. It is not clear to us if we can extend Ashwinkumar's charging scheme to argue that his algorithm can be used in Line 6 of Algorithm 2 . So we give a simpler charging scheme for partition matroids in Appendix $B$ to give better approximation ratios using multiple passes. We get the approximation ratios $p+\varepsilon$ and $p+1+\varepsilon$ for the modular and submodular cases, respectively, by setting $\gamma=\varepsilon /(p+1)$ for both cases, and $\kappa=\gamma^{3} /((p-1)(1+$ $\left.\gamma)^{2}-\gamma^{3}\right)$ for the modular case and $\kappa=\gamma^{3} /\left(p+(2 p-1) \gamma+(p-1) \gamma^{2}-\gamma^{3}\right)$ for the submodular case, in Algorithm 2.

The better, curvature-dependent approximation ratio of Theorem 4 can be obtained using the same idea as for $f$-MSM, by appealing to Lemma 12 and applying it to Ashwinkumar's one-pass algorithm, which, as we have noted, is compliant. 


\section{References}

[1] Kook Jin Ahn and Sudipto Guha. Linear programming in the semi-streaming model with application to the maximum matching problem. In Luca Aceto, Monika Henzinger, and Ji Sgall, editors, Automata, Languages and Programming, volume 6756 of Lecture Notes in Computer Science, pages 526-538. Springer Berlin Heidelberg, 2011.

[2] B. V. Ashwinkumar. Buyback problem: approximate matroid intersection with cancellation costs. In Proceedings of the 38th international colloquium conference on Automata, languages and programming Volume Part I, ICALP'11, pages 379-390, Berlin, Heidelberg, 2011. Springer-Verlag.

[3] B. V. Ashwinkumar and Jan Vondrák. Fast algorithms for maximizing submodular functions. In Proc. 25th Annual ACM-SIAM Symposium on Discrete Algorithms, SODA '14 to appear. SIAM, 2014.

[4] Gruia Calinescu, Chandra Chekuri, Martin Pál, and Jan Vondrák. Maximizing a monotone submodular function subject to a matroid constraint. SIAM J. Comput., 40(6):1740-1766, December 2011.

[5] Jack Edmonds. Paths, trees, and flowers. Canad. J. Math., 17:449-467, 1965.

[6] L. Epstein, A. Levin, J. Mestre, and D. Segev. Improved approximation guarantees for weighted matching in the semi-streaming model. SIAM Journal on Discrete Mathematics, 25(3):1251-1265, 2011.

[7] Joan Feigenbaum, Sampath Kannan, Andrew McGregor, Siddharth Suri, and Jian Zhang. On graph problems in a semi-streaming model. Theor. Comput. Sci., 348(2):207-216, December 2005.

[8] Moran Feldman, Joseph Naor, Roy Schwartz, and Justin Ward. Improved approximations for kexchange systems. In Proc. 19th Annual European Symposium on Algorithms, ESA'11, pages 784-798, Berlin, Heidelberg, 2011. Springer-Verlag.

[9] M.L. Fisher, G.L. Nemhauser, and L.A. Wolsey. An analysis of approximations for maximizing submodular set functions-II. In M.L. Balinski and A.J. Hoffman, editors, Polyhedral Combinatorics, volume 8 of Mathematical Programming Studies, pages 73-87. Springer Berlin Heidelberg, 1978.

[10] Zvi Galil. Efficient algorithms for finding maximum matching in graphs. ACM Comput. Surv., 18(1):2338, 1986.

[11] Ashish Goel, Michael Kapralov, and Sanjeev Khanna. On the communication and streaming complexity of maximum bipartite matching. In Proc. 23rd Annual ACM-SIAM Symposium on Discrete Algorithms, SODA '12, pages 468-485. SIAM, 2012.

[12] T. A. Jenkyns. The efficacy of the "greedy" algorithm. In Proceedings of the 7th South Eastern Conference on Combinatorics, Graph Theory and Computing, pages 341-350, 1976.

[13] Michael Kapralov. Better bounds for matchings in the streaming model. In Proc. 24th Annual ACMSIAM Symposium on Discrete Algorithms, SODA '13. SIAM, 2013.

[14] Richard M. Karp, Umesh V. Vazirani, and Vijay V. Vazirani. An optimal algorithm for on-line bipartite matching. In Proc. 22nd Annual ACM Symposium on the Theory of Computing, pages 352-358, 1990.

[15] Jon Lee, Vahab S. Mirrokni, Viswanath Nagarajan, and Maxim Sviridenko. Non-monotone submodular maximization under matroid and knapsack constraints. In Proc. 41st Annual ACM Symposium on the Theory of Computing, STOC '09, pages 323-332, Bethesda, MD, USA, 2009. ACM.

[16] Jon Lee, Maxim Sviridenko, and Jan Vondrák. Submodular maximization over multiple matroids via generalized exchange properties. Mathematics of Operations Research, 35(4):795-806, November 2010.

[17] Hui Lin and Jeff Bilmes. Word alignment via submodular maximization over matroids. In Proceedings of the 49th Annual Meeting of the Association for Computational Linguistics: Human Language Technologies: short papers - Volume 2, HLT '11, pages 170-175, Stroudsburg, PA, USA, 2011. Association for Computational Linguistics.

[18] Andrew McGregor. Finding graph matchings in data streams. In Proc. 8th International Workshop on Approximation Algorithms for Combinatorial Optimization Problems, APPROX'05/RANDOM'05, pages 170-181, Berlin, Heidelberg, 2005. Springer-Verlag. 
[19] G. L. Nemhauser and L. A. Wolsey. Best algorithms for approximating the maximum of a submodular set function. Mathematics of Operations Research, 3(3):pp. 177-188, 1978.

[20] G.L. Nemhauser, L.A. Wolsey, and M.L. Fisher. An analysis of approximations for maximizing submodular set functions-I. Mathematical Programming, 14(1):265-294, 1978.

[21] Mariano Zelke. Weighted matching in the semi-streaming model. In Proc. 25th International Symposium on Theoretical Aspects of Computer Science, STACS '08, pages 669-680, 2008.

\section{A Details of the Charging Schemes}

We give a self-contained proof of Lemma 16 for the sake of completeness. We also give a generalization of the charging scheme for hypergraphs.

We use the same terminology and notation as in previous sections, but we also denote an edge $\{u, v\} \in E$ as $u v$, for convenience. Matching $M^{*}$ can be thought of as any matching, not just an optimum matching, for this argument. We can charge the weight $w\left(M^{*}\right)$ to the edges in $M \cup K$ in such a way that each edge $e \in K$ gets at most $(1+\gamma) w(e)$ charge and each edge $e \in M$ gets at most $2(1+\gamma) w(e)$ charge. This is essentially the same charging and charge-redistribution scheme as in Feigenbaum et al. [7].

Let $M^{*}=\left\{o_{1} p_{1}, o_{2} p_{2}, \ldots\right\}$. Consider an edge op $\in M^{*}$. If $o p$ was born and killed later by an edge $p q$, then we charge $w(o p)$ to $p q$ and associate this charge with vertex $p$. If $o p$ was born and not killed, then it charges itself $w(o p)$. If $o p$ was not born because of one or two edges $x o, p y \in M \cup K$ then we charge $\frac{w(o p) w(x o)}{w(x o)+w(p y)}$ to $x o$ and associate this charge with vertex $o$ and $\frac{w(o p) w(p y)}{w(x o)+w(p y)}$ to $p y$ and associate this charge with vertex $p$. In all three cases the following holds.

Observation 17. At most $(1+\gamma) w(x y)$ charge is associated with each of $x$ and $y$ for an edge $x y \in M \cup K$.

The redistribution is done as follows. Imagine this happening in the order the edges were processed: whenever an edge $x y$ is killed by an edge $y z$, then we transfer the charge associated with vertex $y$ from edge $x y$ to $y z$. Since $y z$ killed $x y$, we have $w(y z) \geqslant w(x y)$, hence using Observation 17, $y z$ is charged at most $(1+\gamma) w(x y)$ and this charge is associated with vertex $y$ after redistribution, which is at most $(1+\gamma) w(y z)$.

A charge associated with a vertex $v$ is from an edge, say $u v \in M^{*}$. Hence, after this redistribution, each edge in $K$ has charge associated with at most one vertex, and each edge in $M$ has charge associated with at most two vertices, and this completes the proof.

\section{A.1 Charging Scheme for Hypergraphs}

The charging and redistribution argument is generalized as follows. Recall that an edge $e$ in a hypergraph is a subset of the set of vertices, i.e., if $e \in E$, then $e \subseteq V$.

Here, we charge the weight $w\left(M^{*}\right)$ to the edges in $M \cup K$ such that each edge $e \in K$ gets at most $(p-1)(1+\gamma) w(e)$ charge and each edge $e \in M$ gets at most $p(1+\gamma) w(e)$ charge. Consider an edge $e^{*} \in M^{*}$. If $e^{*}$ was born and killed later, then we charge $w\left(e^{*}\right)$ to its murderer $e^{\dagger}$ and associate this charge with vertices in $e^{*} \cap e^{\dagger}$. If $e^{*}$ was born and not killed, then it charges itself. If $e^{*}$ is not born because of at most $p$ edges, say $e_{1}, \ldots, e_{p}$, then we charge $w\left(e_{i}\right) w\left(e^{*}\right) / \sum_{i} w\left(e_{i}\right)$ to edge $e_{i}$ and associate these charges with vertices $e^{*} \cap e_{i}$ correspondingly. In all the cases, an edge $e$ is charged at most $(1+\gamma) w(e)$ by a given edge in $M^{*}$, and thus bears at most $p(1+\gamma)$ charge.

For redistribution, when an edge $e^{\prime}$ is killed by $e^{k}$, we transfer all the charge associated with vertices $e^{\prime} \cap e^{k}$ to $e^{k}$. For example, if an edge $e=\{a, b, c, d\}$ did not let edges $\{a, b\}$ and $\{c\}$ be born, then $e$ bears $w(\{a, b\})$ charge associated with $a$ and $b$, and $w(\{c\})$ charge associated with $c$. If in the 
future $\{a\}$ kills $e$, then we transfer $w(\{a, b\})$ charge to $\{a\}$, or if $\{a, c, x, y\}$ kills $e$, then we transfer $w(\{a, b\})+w(\{c\})$ charge from $e$ to $\{a, c, x, y\}$. Thus, an edge $e$ not in the final matching bears at most $(p-1)(1+\gamma) w(e)$ charge, and an edge $e$ in the final matching bears at most $p(1+\gamma) w(e)$ charge. This gives us Equation (8)).

\section{B Ashwinkumar's Algorithm and a New Charging Scheme}

We give Ashwinkumar's Algorithm for $p$ matroids and a simpler charging scheme for partition matroids.

Let $\left(E^{1}, \ldots, E^{r}\right)$ be a partition of $E$, and $k^{1}, \ldots, k^{r}$ be positive integers, and

$$
\mathcal{I}=\left\{I \subseteq E:\left|I \cap E^{j}\right| \leqslant k^{j} \text { for all } j \in[r]\right\},
$$

then $(E, \mathcal{I})$ is a matroid, specifically, a partition matroid. Also, a set that is not independent is dependent, and a minimally dependent set is a circuit. We say that a set $J \subseteq E$ saturates $E^{s}$ if $\left|J \cap E^{S}\right|=k^{S}$. We denote the partition of matroid $M_{i}$ by $E_{i}^{1}, \ldots$, and corresponding constraints by $k_{i}^{1}, \ldots$. For an independent set $I \in \mathcal{I}_{i}$ and an element $e \in E-I$ such that $I+e \notin \mathcal{I}_{i}$, let $C_{i}(I+e)$ denote the unique circuit in $I+e$. Now, consider Algorithm 3 .

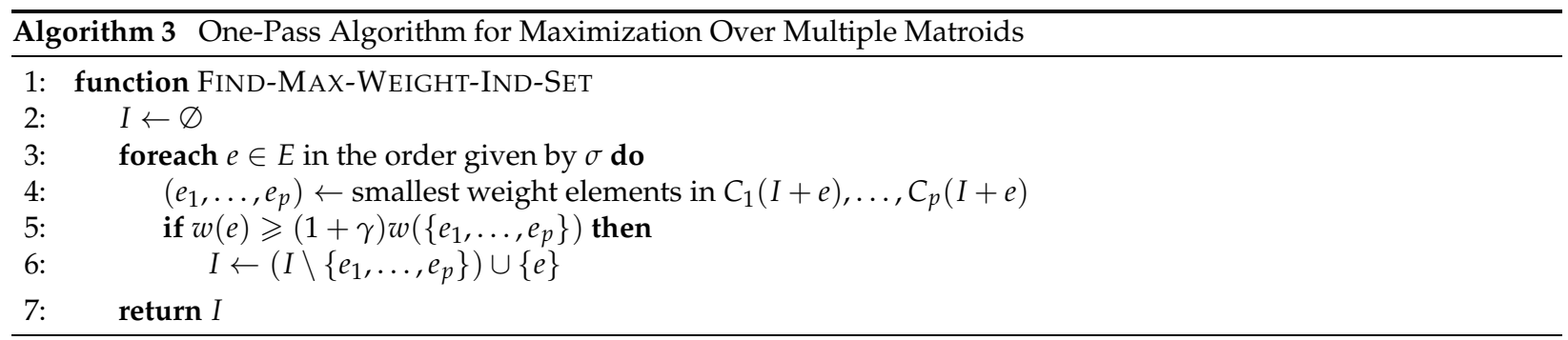

\section{B.1 A Simpler Charging Scheme For Partition Matroids}

Let $I^{*}$ be an optimal independent set and $I$ be the output. We reuse some of the terminology used in Section 4. When an element $e$ is added to $I$ in Line 6, we say that $e$ is born and that it kills the (at most $p$ ) elements $e_{1}, \ldots, e_{p}$. Let $I_{e}$ denote the maintained independent set just before $e$ was processed. Let $K=\left(\bigcup_{e \in E} I_{e}\right) \backslash I$ denote the set of elements that were added to the maintained independent set at some point but did not make it to the final output. These elements can be made the nodes of a collection of disjoint rooted killing trees where the parent of a killed element $e$ is the edge $e^{\prime}$ that killed it. The set of roots of these killing trees is precisely $I$. Let $T(e)$ denote the set of strict descendants of $e \in I$ in its killing tree. Then $K=\bigcup_{e \in I} T(e)$.

We give a charging scheme that is based on the one mentioned in the proof of Lemma 16 but is slightly different from that used in case of hypergraphs. We charge responsible elements with respect to a matroid, i.e., if $e \in I^{*}$ charges $e^{\prime} \in I_{e}$ because $I_{e}+e$ formed a circuit in some $M_{i}$, then we associate this charge to $e^{\prime}$ with $M_{i}$. Now, if $e \in I^{*}$ is taken, then it charges itself and this charge is associated with all $p$ matroids. Suppose $e \in I^{*}$ was not taken because of the elements $e_{1}^{j_{1}}, \ldots, e_{p}^{j_{p}}$ (see Line 4 ), where $j_{1}, \ldots, j_{p}$ denote the set indices in partitions of $M_{1}, \ldots, M_{p}$. Then for all $e^{\prime} \in\left(\left(C_{1}\left(I_{e}+e\right)-e\right) \cup \cdots \cup\left(C_{p}\left(I_{e}+e\right)-e\right)\right)$, we have $w(e)<(1+\gamma) w\left(e^{\prime}\right)$. Also, $e \in E_{i}^{j_{i}}$ for $i \in[p]$ and some corresponding $j_{i}$. Since $I_{e}$ saturates $E_{i}^{j_{i}}$ for all $i \in[p]$, there exist elements $e_{1}^{\prime} \in\left(C_{1}\left(I_{e}+e\right)-e\right), \ldots, e_{p}^{\prime} \in\left(C_{p}\left(I_{e}+e\right)-e\right)$, such that $e_{1}^{\prime}, \ldots, e_{p}^{\prime}$ were uncharged with respect to 
$M_{1}, \ldots, M_{p}$, respectively, because for all $i \in[p]$, we have $\left|I^{*} \cap E_{i}^{j_{i}}\right| \leqslant k_{i}^{j_{i}}$. So, for $i \in[p]$, we charge $w\left(e_{i}^{\prime}\right) w(e) /\left(\sum_{j=1}^{p} w\left(e_{j}^{\prime}\right)\right)$ to $e_{i}^{\prime}$. In any case, an element $e^{\prime}$ is charged at most $(1+\gamma) w\left(e^{\prime}\right)$ and by at most one optimal element with respect to a particular matroid, i.e., $p(1+\gamma) w\left(e^{\prime}\right)$ in total.

The charge redistribution is as follows. Whenever elements $e_{1}^{j_{1}}, \ldots, e_{p}^{j_{p}}$ are killed by $e^{k}$, we transfer the charge on $e_{1}^{j_{1}}, \ldots, e_{p}^{j_{p}}$ associated with $M_{1}, \ldots, M_{p}$, respectively, to $e^{k}$ keeping the same association, and the amount of charge transferred is at most $(1+\gamma) w\left(e^{k}\right)$. Note that after this transfer, if an $e^{k}$ carried a charge associated with some $M_{i}$, then it will not be charged again for that matroid. Thus, after all the redistribution, an element $e^{\prime} \in K$ carries at most $(p-1)(1+\gamma) w\left(e^{\prime}\right)$ charge, and an element $e^{\prime} \in I$ carries at most $p(1+\gamma) w\left(e^{\prime}\right)$ charge. So we get an inequality similar to Equation (8) for hypergraphs,

$$
w\left(I^{*}\right) \leqslant \sum_{e \in I}(1+\gamma)((p-1) w(T(e))+p w(e)) .
$$

Now, Equation (9) enables us to extend Algorithm 3 to the multi-pass version based on McGregor's algorithm for MWM, and its $f$-extension, i.e., Algorithm 2, and we get the approximation ratios $p+\varepsilon$ and $p+1+\varepsilon$ for modular and submodular case, respectively, by setting $\gamma=\varepsilon /(p+1)$ for both linear and submodular case, and $\kappa=\gamma^{3} /\left((p-1)(1+\gamma)^{2}-\gamma^{3}\right)$ for linear case and $\kappa=\gamma^{3} /\left(p+(2 p-1) \gamma+(p-1) \gamma^{2}-\gamma^{3}\right)$ for submodular case.

\section{Zelke's Algorithm for MWM}

As mentioned earlier, Zelke's algorithm is compliant. Therefore, to describe its logic in full, it suffices to explain what happens in Lines 8 to 9 .

For each edge $u v \in M$, the algorithm stores at most two shadow edges associated with each of $u$ and $v$, denoted by shadow-edge $(u v, u)$ and shadow-edge $(u v, v)$. When an edge $y_{1} y_{2}$ arrives in the stream, it considers the following set of at most seven edges in the vicinity of $y_{1} y_{2}$, viz., $T=\left\{y_{1} y_{2}, g_{1} y_{1}, a_{1} g_{1}, a_{1} c_{1}, g_{2} y_{2}, a_{2} g_{2}, a_{2} c_{2}\right\}$, where

- $g_{1} y_{1}, g_{2} y_{2}$ are edges in $M$ that intersect with $y_{1} y_{2}$,

- $a_{1} g_{1}=$ shadow-edge $\left(g_{1} y_{1}, g_{1}\right)$ and $a_{2} g_{2}=$ shadow-edge $\left(g_{2} y_{2}, g_{2}\right)$,

- $a_{1} c_{1}$ and $a_{2} c_{2}$ are the edges in $M$ that intersect with $a_{1} g_{1}$ and $a_{2} g_{2}$, respectively.

For a set $A \subseteq E$ and a matching $M \subseteq E$, let $M\ulcorner A$ denote the set of edges in $M$ that share a vertex with some edge in $A$. The algorithm picks an augmenting set $A \subseteq T$ that is a matching and maximizes the difference $w(A)-(1+\gamma) w(M 「 A)$. If this difference is positive, then $A$ is chosen in Line 8 , i.e., the matching $M$ is updated as follows:

$$
M \leftarrow(M \backslash(M\ulcorner A)) \cup A .
$$

Then the set of shadow edges $S$ is updated as

$$
S \leftarrow\left(S \backslash\left(A \cup \bigcup_{u v \in M \sqcap A}\{\operatorname{shadow-edge}(u v, u), \operatorname{shadow} \text {-edge }(u v, v)\}\right)\right) \cup(M\ulcorner A) ;
$$

note that since we are removing shadow edges of the edges in $M\ulcorner A$, the number of shadow edges remains at most $n$. The edges in $M \gg A$ then become shadow edges associated with the vertices that it shares with the edges in $M$. For example, if $A=\left\{a_{1} g_{1}\right\}$, then $M \gg A=$ $\left\{g_{1} y_{1}, a_{1} c_{1}\right\}$, and after updating $M$ and $S$ as mentioned above, shadow-edge $\left(a_{1} g_{1}, g_{1}\right)=g_{1} y_{1}$, and shadow-edge $\left(a_{1} g_{1}, a_{1}\right)=a_{1} c_{1}$. This completes the description of Zelke's algorithm. 